\title{
B-cell translocation 1 gene inhibits cellular metastasis-associated behavior in breast cancer
}

\author{
WEI LI ${ }^{1 *}$, SHI-TAO ZOU ${ }^{2 *}$, RAN ZHU $^{3}$, JIAN-MEI WAN ${ }^{3}$, YAN XU $^{4}$ and HAO-RONG WU ${ }^{1}$ \\ ${ }^{1}$ Department of General Surgery, Second Affiliated Hospital of Soochow University, Suzhou, Jiangsu 215004; \\ ${ }^{2}$ Oncology Institute, Wuxi Fourth People's Hospital, Wuxi, Jiangsu 214062; ${ }^{3}$ School of Radiation Medicine and Protection, \\ Medical College, Soochow University; ${ }^{4}$ Advanced Health Center, The First Affiliated Hospital of \\ Soochow University, Suzhou, Jiangsu 215123, P.R. China
}

Received August 30, 2013; Accepted March 6, 2014

DOI: $10.3892 / \mathrm{mmr} .2014 .2118$

\begin{abstract}
B-cell translocation gene 1 (BTG1) is a member of the BTG/transducer of ERBB2 family, which regulates cell cycle progression in a variety of cell types and may have a role in inhibiting proliferation, promoting apoptosis and stimulating cellular differentiation in numerous cell types. However, the role of BTG1 in cancer metastasis is yet to be elucidated. In the present study, analysis of clinical specimens revealed that BTG1 mRNA levels were lower in lymph node metastases than those in benign breast tumors and normal human breast tissue. The effect of BTG1 on the metastatic behavior of breast cancer cells following stable transfection with a BTG1 expression vector was also investigated. The overexpression of BTG1 was observed to inhibit cell adhesion, migration and invasion. Furthermore, the overexpression of BTG1 was found to be involved in the inhibition of the metastasis-related proteins matrix metalloproteinase- 2 and -9 , as well as the promotion of the cell-cell adhesion-associated protein E-cadherin. In syngeneic nude mice breast tumor models, hepatic metastasis and angiogenesis were observed in the mice injected with the control cells, but not in those injected with pcDNA3-BTG1 cells. Immunohistochemistry revealed that overexpression of BTG1 decreased vascular endothelial growth factor expression in tumors. To the best of our knowledge, this is the first study to show that BTG1 overexpression decreases migration and invasion of breast cancer cells and thereby inhibits distant metastasis in mice breast tumor models.
\end{abstract}

\footnotetext{
Correspondence to: Professor Hao-Rong Wu, Department of General Surgery, Second Affiliated Hospital of Soochow University, 181 Sanxiang Road, Suzhou, Jiangsu 215004, P.R. China

E-mail: sdzoushitao@126.com

${ }^{*}$ Contributed equally
}

Key words: BTG1, breast cancer cell, metastasis, invasion, MMPs

\section{Introduction}

Tumor metastasis is a significant clinical problem, which complicates therapeutic cancer treatment and is responsible for $\sim 90 \%$ of all cancer mortalities $(1,2)$. The clinical efficacy of therapeutic modalities is impaired by the presence of metastatic tumors. In females, breast cancer accounts for $\sim 23 \%$ of all diagnosed cancer cases, which is the highest among all types of cancer and accounts for 7.6 million mortalities annually worldwide (3). Early tumor metastasis to different organs is one of the major causes of low survival rates in patients with breast cancer $(2,4,5)$.

B-cell translocation gene 1 (BTG1) is a member of the anti-proliferative gene family comprising pheochromacytoma cell-3, tetradecanoyl phorbol acetate-inducible sequence 21, BTG3, transducer of ERBB2, 1 (TOB1) and TOB2 $(6,7)$. BTG1 was originally identified as a sequence associated with a chromosomal translocation in a lymphoid malignancy $(6,8)$. Proteins encoded by members of this gene family have been implicated in the induction of growth arrest or apoptosis in a variety of cell systems (8). Overexpression of BTG1 has been found to inhibit proliferation during normal erythroid differentiation (9) and to induce growth arrest in a B-cell lymphoma model (10). Furthermore, exogenous expression of BTG1 has been found to reduce proliferation and induce $\mathrm{G}_{1}$ arrest and/or apoptosis in several cell types, including NIH3T3 murine fibroblasts (11), microglia (12) and myoblasts (13). A role for BTG1 in cellular differentiation has also been proposed based on investigations showing that BTG1 expression stimulates myoblast differentiation (13), and that BTG1 is upregulated in leukemic cells upon treatment with chemicals that induce differentiation (14).

BTG1 may therefore have a role in inhibiting proliferation, promoting apoptosis and stimulating cellular differentiation in multiple cell types. However, the role of BTG1 in cancer metastasis is yet to be elucidated. In the present study, the effect of BTG1 on breast cancer cell adhesion, migration and invasion was investigated. In addition, breast cancer distant metastasis and angiogenesis were also investigated in a nude mouse tumor model. To the best of our knowledge, this is the first study to show that overexpression of BTG1 inhibits breast cancer metastasis. 


\section{Materials and methods}

Cell culture. Human breast cancer cell lines, MCF-7 and MDA-MB-231, were obtained from the Shanghai Cell Bank (Shanghai, China). Cells were cultured in Dulbecco's Modified Eagle's medium (Gibco-BRL, Carlsbad, CA, USA) containing $10 \%$ fetal bovine serum in a humidified atmosphere with $5 \% \mathrm{CO}_{2}$ at $37^{\circ} \mathrm{C}$. 2. The present study was approved by the ethics committee of Soochow University (Suzhou, Jiangsu, China).

Generation of stable cell lines. In order to generate stable cell lines overexpressing BTG1, cDNAs for full-length human BTG1 were amplified by the polymerase chain reaction (PCR) with the following primer sequences: 5'-CACCATGCA TCCCTTCTACACCCGG-3' (forward) and 5'-TTAACCTGA TACAGTCATCATATTG-3' (reverse). The full-length cDNA was then cloned into a XhoI and BamHI linearized plasmid vector, pcDNA3 (Clontech Laboratories, Mountain View, CA, USA). The control pcDNA3 vector (pcDNA3-neo) or the human BTG1 expression vector, pcDNA3-BTG1, were transfected into MCF-7 or MDA-MB-231 cells using Lipofectamine 2000 (Invitrogen Life Technologies, Carlsbad, CA, USA). Stable clones were selected in medium containing $500 \mu \mathrm{g} / \mathrm{ml}$ G418 (Sigma-Aldrich, St. Louis, MO, USA). Individual clones were isolated and expanded for further characterization. The expression of BTG1 was determined using reverse transcription (RT)-PCR and western blot analyses.

Cell adhesion assay. A flat-bottomed 96-well plate was coated

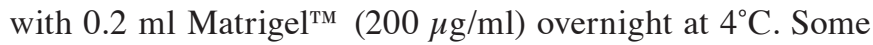
wells were left uncoated as negative controls. The plate was washed twice with phosphate-buffered saline (PBS) and blocked with $1 \mathrm{mg} / \mathrm{ml}$ bovine serum albumin (BSA) for $2 \mathrm{~h}$ at $37^{\circ} \mathrm{C}$, prior to the addition of $0.5 \mathrm{ml}\left(5 \times 10^{3}\right)$ cells, which were transfected as described above. Following incubation at $0.5-, 1-$, 1.5- and 2-h intervals at $37^{\circ} \mathrm{C}$, unattached cells were removed from the plate by washing with PBS. MTT (Sigma-Aldrich) was added to each well and the percentage adhesion was calculated by dividing the absorbance values obtained from the coated wells by the absorbance values obtained from the uncoated wells (100\%).

Wound-healing assay in vitro. A wound-healing assay was used to detect cell migration in vitro. Cells were seeded in six-well plates at a density of $1 \times 10^{6}$ cells/well and incubated at $37^{\circ} \mathrm{C}$ for $24 \mathrm{~h}$. When cells were $90 \%$ confluent, a thin mark was drawn vertically with a pipette tip in the six-well plate. Cells were then washed three times with sterile PBS to remove any unattached cells. Following incubation for 24 or $48 \mathrm{~h}$ at $37^{\circ} \mathrm{C}$ with $5 \% \mathrm{CO}_{2}$, wound width was assessed using light microscopy.

Cell invasion and migration assay. Cell invasion was measured using Matrigel-coated Transwell ${ }^{\circledR}$ inserts (Costar Inc., Cambridge, UK). Cells were initially cultured in serum-free medium for $12-24 \mathrm{~h}$. A total of $5 \times 10^{4}(100 \mu \mathrm{l})$ cells were then plated in serum-free medium with BSA on 24-well Transwell inserts coated with $100 \mathrm{ml}$ Matrigel $(250 \mathrm{mg} / \mathrm{ml})$. The underside of the insert was precoated with
$500 \mu \mathrm{l}$ chemokine-containing cell supernatant. Following incubation for $48 \mathrm{~h}$ at $37^{\circ} \mathrm{C}$ with $5 \% \mathrm{CO}_{2}$, the inserts were fixed with $3.7 \%$ paraformaldehyde/PBS and stained with $2 \%$ crystal violet. The penetration of cells through the membrane was quantified by counting the number of cells that penetrated the membrane in 10 microscopic fields/filter (magnification, $\mathrm{x} 200$ ). The experiment was repeated three times. Cell migration assays were performed in a similar manner, but without the Matrigel coating.

Western blot analysis. Each experimental group of cells was lysed using SDS sample buffer (80 mM Tris-HCl, 2\% SDS, $300 \mathrm{mM} \mathrm{NaCl}$ and $1.6 \mathrm{mM}$ EDTA). Cell extracts were separated using $10 \%$ SDS-PAGE, transferred onto nitrocellulose membranes and blocked with 5\% skimmed milk. Following blocking, membranes were incubated with antibodies against $\beta$-actin, BTG1, matrix metalloproteinase (MMP)-2, MMP-9 and E-cadherin (E-cad) (Santa Cruz Biotechnology, Inc., Santa Cruz, CA, USA), and then incubated with horseradish peroxidase (HRP)-conjugated anti-mouse or -rabbit immunoglobulin $\mathrm{G}$ antibodies (Santa Cruz Biotechnology, Inc.). Protein bands were visualized using enhanced chemiluminescence solution.

In vivo studies. Four-week old female nude mice (specific pathogen-free BALB/c) were obtained from the Laboratory Animal Center of Soochow University (Suzhou, China) and housed at a constant temperature $\left(23 \pm 2^{\circ} \mathrm{C}\right)$ and humidity (50-70\%) with a 12-h light/dark cycle. MDA-MB-231 cells were either transfected with pcDNA3-BTG1 or pcDNA3-neo (negative control), or left untreated (blank), as described previously. Cells were grown to subconfluency, trypsinized, harvested, washed twice with PBS and resuspended in $0.2 \mathrm{ml}$ PBS $\left(5 \times 10^{6}\right.$ cells $\left./ 0.2 \mathrm{ml}\right)$, prior to subcutaneous injection into the three groups of mice. Each group contained six nude mice. Five weeks after injection, the presence of metastases in the lung and liver tissues from each mouse bearing a tumor mass on the back was evaluated by gross and microscopic examination. Tissue sections were deparaffinized, rehydrated and rinsed, prior to hematoxylin and eosin staining and examination for metastatic nodules and cell pathology.

Immunohistochemistry. Tumor xenografts were removed, deparaffinized, rehydrated, washed and subjected to antigen retrieval. Following washing in distilled water and PBS, xenografts were treated with $0.03 \%$ hydrogen peroxide for 5 min to block endogenous peroxidase activity. Xenografts were then incubated with mouse anti-human cluster of differentiation (CD) 31, BTG1 and vascular endothelial growth factor (VEGF) antibodies (Santa Cruz Biotechnology Inc.) diluted 1:100 for $60 \mathrm{~min}$ at room temperature. Following washing in PBS, the sections were incubated with labeled HRP-conjugated anti-mouse antibodies, for $30 \mathrm{~min}$ at room temperature, prior to washing twice in PBS and incubating with diaminobenzene for $10 \mathrm{~min}$. Following washing, the sections were counterstained with hematoxylin, washed and dipped briefly in a water bath containing drops of ammonia, prior to dehydration and mounting in Diatex. The stained sections were analyzed and scored using a Nikon microscope (Nikon Corp., Tokyo, Japan). 
A
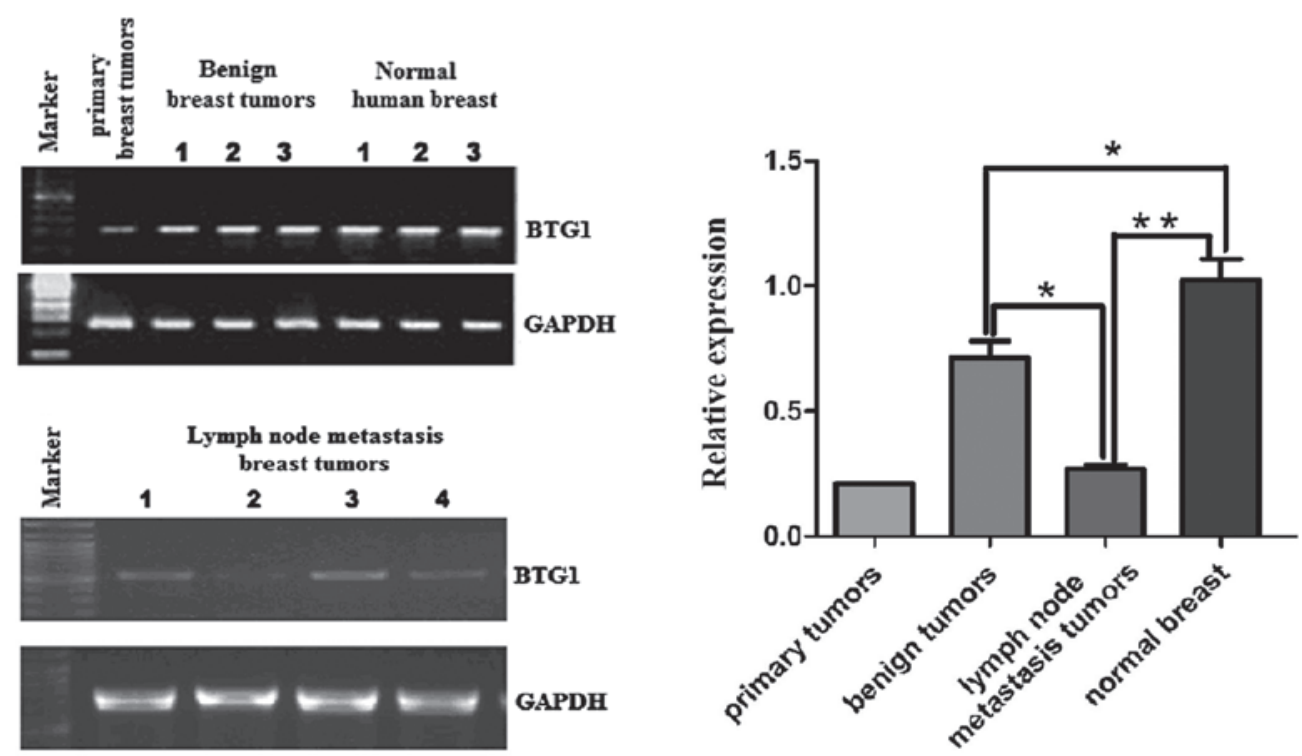

B
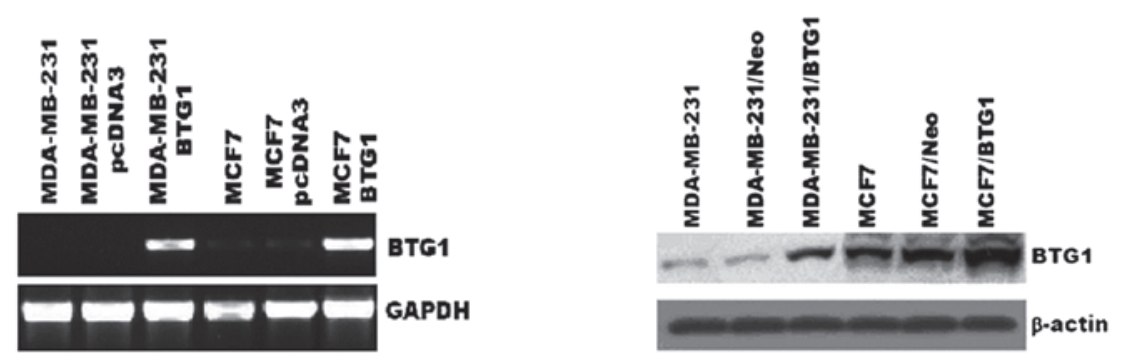

Figure 1. BTG1 expression in clinical specimens and the generation of stable cell lines. (A) BTG1 mRNA was detected using RT-PCR in primary tumors, lymph node metastases, benign breast tumors and normal human breast tissue, as indicated. GAPDH levels were used as a loading control. BTG1 mRNA levels in normal human breast tissue were significantly higher than those in benign breast tumors and lymph node metastases $\left({ }^{*} \mathrm{P}<0.05,{ }^{, *} \mathrm{P}<0.01\right)$. (B) RT-PCR and western blot analysis showing BTG1 mRNA and protein levels in MCF-7 and MDA-MB-231 cells transfected with either control pcDNA3-neo or pcDNA3-BTG1 plasmids and their corresponding untreated cells. GAPDH and $\beta$-actin levels were used as loading controls for the RT-PCR and western blotting, respectively. RT-PCR, reverse transcription polymerase chain reaction; BTG1, B-cell translocation gene 1.

Statistical analysis. Results are presented as the mean \pm standard deviation. A value of $\mathrm{P}<0.05$ was considered to indicate a statistically significant difference. Statistical analyses were performed using SPSS 17.0 (SPSS Inc., Chicago, IL, USA). Each experiment was repeated three times.

\section{Results}

BTG1 expression in clinical specimens and establishment of BTG1 overexpression in breast cancer cell lines. RT-PCR was used to assess the expression of BTG1 in human breast tissues from primary tumors, lymph node metastases, benign breast tumors and normal human breast tissue (Fig. 1A). BTG1 mRNA levels were observed to be significantly higher in normal human breast tissue than those in benign breast tumors and lymph node metastases $(\mathrm{P}<0.05)$. Compared with lymph node metastases, BTG1 mRNA levels were found to be significantly higher in benign breast tumors $(\mathrm{P}<0.05)$.

To further explore the role of BTG1 in breast cancer, MCF-7 and MDA-MB-231 cells were used to stably overexpress BTG1 (MDA-MB-231/BTG1 or MCF-7/BTG1). As shown in Fig. 1B, BTG1 mRNA and protein levels were examined in the MCF-7 and MDA-MB-231 cells using RT-PCR and western blot analysis. When compared with the blank or pcDNA3-neo cells, BTG1 expression was significantly increased in the pcDN3-BTG1-transfected cells.

BTG1 decreases cell adhesion in breast cancer cell lines. To assess whether BTG1 expression is associated with adhesion in breast cancer cells, an in vitro adhesion assay was performed to evaluate the adhesive capacity of the blank, pcDNA3-neo and pcDNA3-BTG1 cells. As shown in Fig. 2A, the rate of cell adhesion in the pcDNA3-BTG1 group was significantly decreased compared with that in the blank and pcDNA3-neo groups of cells $(\mathrm{P}<0.05)$.

BTG1 decreases migratory and invasive capacity in breast cancer cell lines. To examine whether overexpression of BTG1 decreases migratory and invasive capacity in breast cancer cell lines, wound healing, migration and invasion assays were performed in MDA-MB-231 and MCF-7 cells. The wound healing assay revealed that blank and pcDNA3-neo cells demonstrated more rapid wound closure than pcDNA3-BTG1 cells (Fig. 2B). Furthermore, as shown in Fig. 3A, cell invasion assay revealed that the number of invading cells significantly decreased with BTG1 overexpression $(\mathrm{P}<0.05)$. It was also observed that the BTG1-overexpressing pcDNA3-BTG1-transfected cells exhibited significantly reduced motility towards 
A

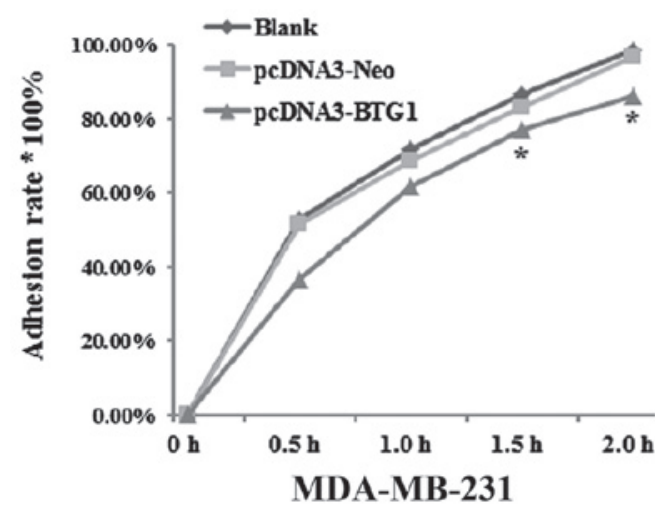

B

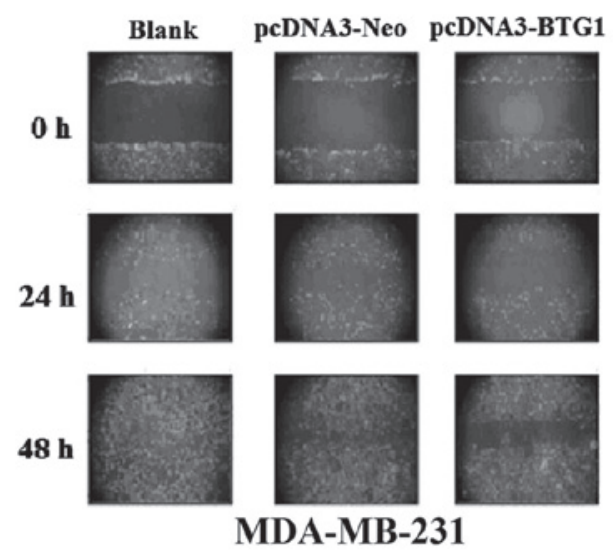

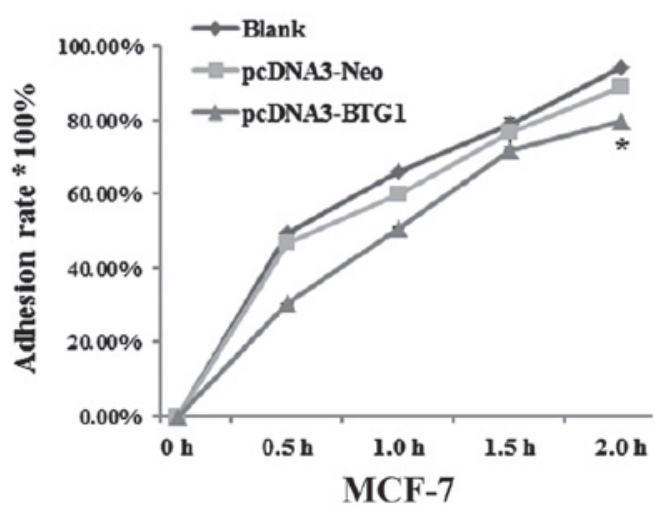

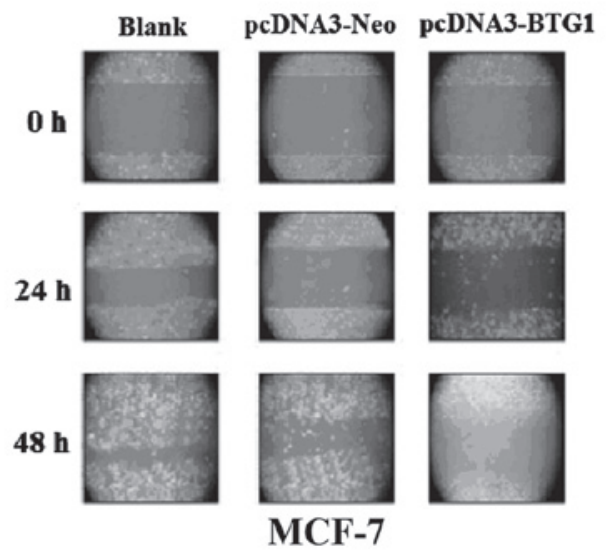

Figure 2. BTG1 attenuates cell adhesion and migration in breast cancer cells. (A) Effect of BTG1 overexpression on MCF-7 and MDA-MB-231 cell adhesion. Three groups of cells were seeded on 96 -well plates, blocked with $1 \mathrm{mg} / \mathrm{ml}$ bovine serum albumin and incubated at $37^{\circ} \mathrm{C}$ for $0.5,1,1.5$ and $2 \mathrm{~h}$. Cells were then counted using the MTT assay ("P $<0.05$ vs. blank and pcDNA3-neo cells). (B) Effect of BTG1 overexpression on MCF-7 and MDA-MB-231 cell migration Cell motility was assessed using a wound healing assay, which showed that wound closure was more rapid in blank cells or pcDNA3-neo cells than that in pcDNA3-BTG1 cells. BTG1, B-cell translocation gene 1.

chemokines compared with that in blank and pcDNA3-neo breast cancer cells (Fig. 3B; $\mathrm{P}<0.05$ ).

Western blot analysis was performed for detection of the metastasis-associated proteins MMP-2 and -9 and the cell-cell adhesion-associated protein E-cad. As shown in Fig. 3C, overexpression of BTG1 was observed to significantly inhibit MMP-2 and -9 expression $(\mathrm{P}<0.05)$ and significantly induce E-cad expression $(\mathrm{P}<0.05)$.

In summary, these results suggest that BTG1 expression is inversely associated with the invasiveness of breast cancer cells in vitro. The inverse correlation between BTG1 expression and invasive capacity in breast cancer cells in vitro indicates that BTG1 may be a metastasis suppressor gene in breast cancer cells.

BTG1 negatively affects distant tumor metastasis and angiogenesis in a nude mouse tumor model in vivo. Three groups of MDA-MB-231 cells were subcutaneously injected into nude mice. Five weeks after injection, the effect of BTG1 overexpression on the promotion of distant metastases was assessed in a mouse tumor model. Lung and liver tissue samples were obtained from the blank $(n=6)$, pcDNA3-neo $(n=6)$ and pcDNA3-BTG1 $(n=6)$ groups of mice. Hepatic metastases were observed in the liver tissue in one out of six mice from the blank and pcDNA3-neo groups (Fig. 4A). However, no significant hepatic metastases were found in the mice in the
pcDNA3-BTG1 group, and no pulmonary metastases were observed in any of the three groups.

The effect of BTG1 overexpression on angiogenesis in tumors in vivo was then assessed using immunohistochemistry. Anti-CD31 antibodies were utilized to detect CD31 levels, which were used to reflect the level of vascular endothelial proliferation. As shown in Fig. 4B, the number of tumor microvessels in the blank and pcDNA3-neo tumor groups, was $28.33 \pm 1.53$ and $29.67 \pm 2.08$, respectively. By contrast, the number of tumor microvessels in the pcDNA3-BTG1 group was $14.00 \pm 1.00$. Furthermore, in the tumors in the pcDNA3-BTG1 group, BTG1 expression was observed to be higher (Fig. 4C) and the level of VEGF expression was observed to be lower than that in the blank and pcDNA3-neo groups. These results suggest that overexpression of BTG1 may be capable of inhibiting angiogenesis through the downregulation of VEGF expression, thereby inhibiting tumor metastasis.

\section{Discussion}

Metastasis is the leading cause of mortality in patients with cancer, and strategies to inhibit tumor cell invasion are a major focus of current efforts to develop cancer treatments. In the present study, the role of BTG1 in breast cancer metastasis was investigated. Analysis of clinical specimens showed that BTG1 mRNA levels were lower in lymph node metastases than 
A

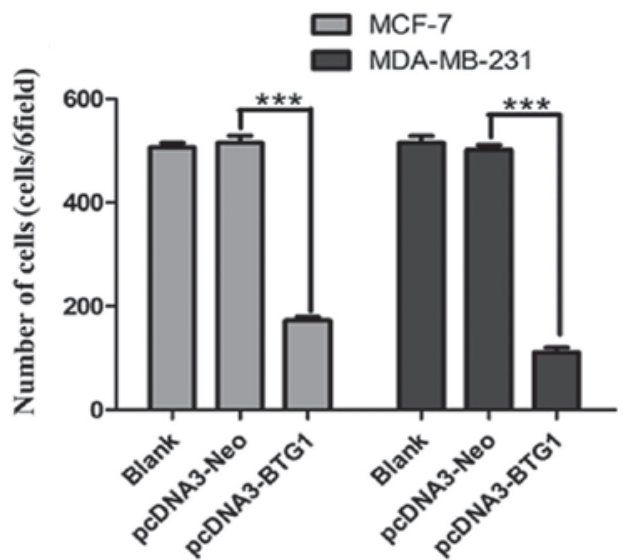

C
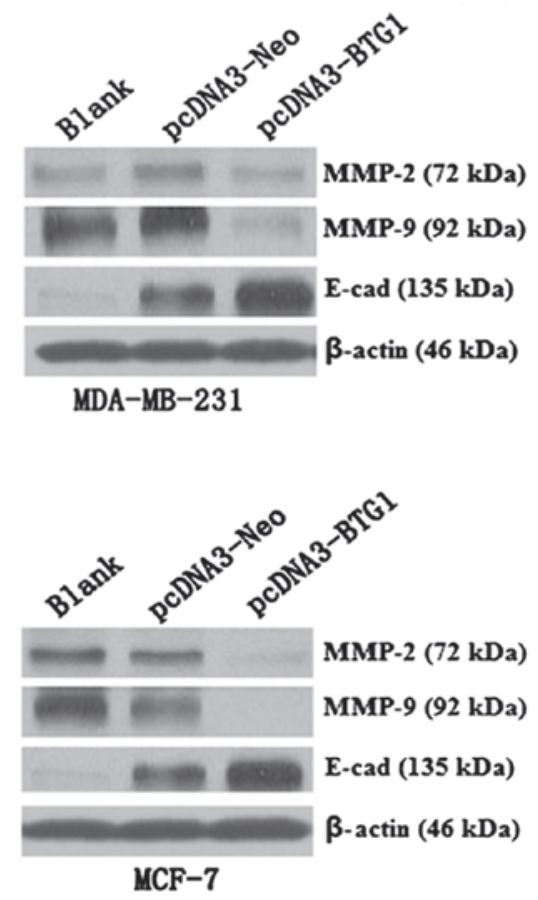

B
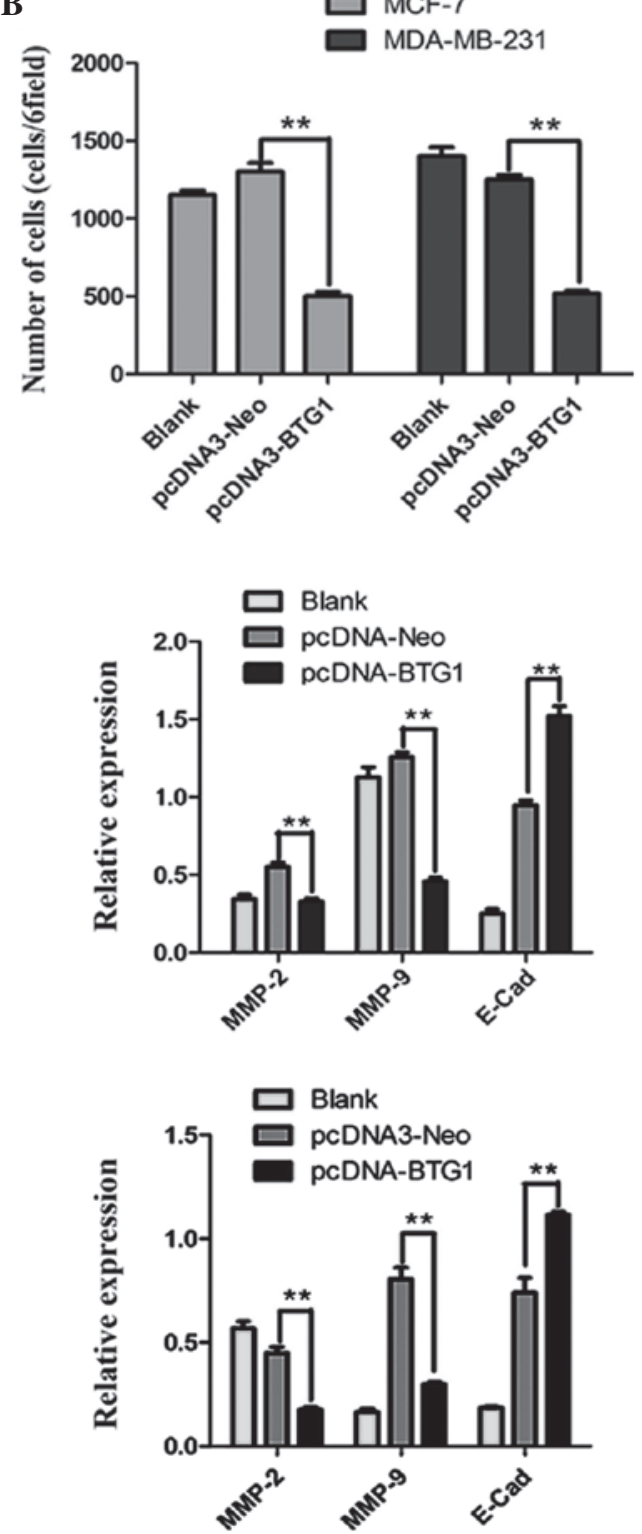

Figure 3. BTG1 attenuates invasive capacity and affects metastasis-related protein expression in breast cancer cell lines. (A) Effect of BTG1 overexpression on MCF-7 and MDA-MB-231 cell invasion. Invasive capacity was determined by counting the cells that penetrated through the membrane. The number of invading cells decreased with BTG1 overexpression ( $\left.{ }^{* * *} \mathrm{P}<0.001\right)$. (B) BTG1-overexpressing MCF-7 and MDA-MB-231 cells transfected with pcDNA3-BTG1 exhibited significantly reduced motility towards chemokines compared with blank and pcDNA3-neo MCF-7 and MDA-MB-231 cells ( $\left.{ }^{* *} \mathrm{P}<0.01\right)$. (C) Overexpression of BTG1 inhibited MMP-2 and -9 expression ( $\left.{ }^{* *} \mathrm{P}<0.01\right)$, and promoted E-cad expression ( $\left.{ }^{* *} \mathrm{P}<0.01\right)$, as shown using western blot analysis. BTG1, B-cell translocation gene 1; MMP, matrix metalloproteinase; E-cad, E-cadherin.

those in benign breast tumors and normal human breast tissue. BTG1 expression vector-induced overexpression of BTG1 mRNA and protein in breast cancer cells was found to inhibit cell adhesion, migration and invasion, as well as in vivo distant tumor metastasis and angiogenesis in a nude mouse tumor model. The findings of the present study show that BTG1 may have a role in breast cancer metastasis. Furthermore, in an attempt to elucidate the mechanism underlying the observed effects, the regulation of key cell invasion genes by BTG1 was investigated.

The metastatic process is composed of the following sequential steps: (i) Tumor cell detachment from the primary neoplasm and invasion of the extracellular matrix (ECM); (ii) intravasation of the hematogenous or lymph system; (iii) arrest in a secondary organ site; (iv) extravasation from the vessel of delivery and (v) proliferation in the newly defined site (15). The molecular mechanisms underlying each of these steps have been described previously (16-18). Novel anti-metastasis therapies are being developed to inhibit these processes.

The formation of transient attachments is necessary for tumor cells to metastasize. Metastasizing tumor cells must be able to attach to ECM components and other cells (19). In the present study, a cell adhesion assay revealed that overexpression of BTG1 decreased the adhesive capacity of breast cancer cells in vitro. Furthermore, a significant decrease in motility was observed in the pcDNA3-BTG1-transfected breast cancer cells overexpressing BTG1.

Tumor cell invasion is the active process of the translocation of neoplastic cells across ECM barriers. Invasion requires 
A

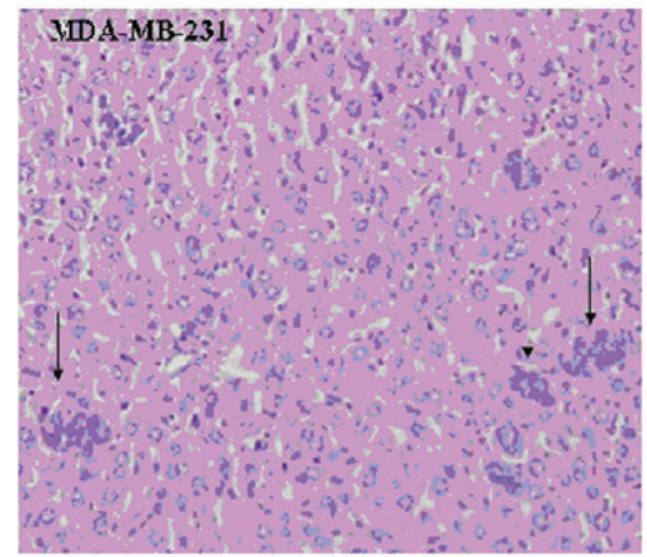

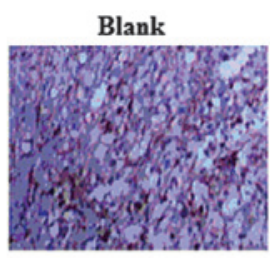
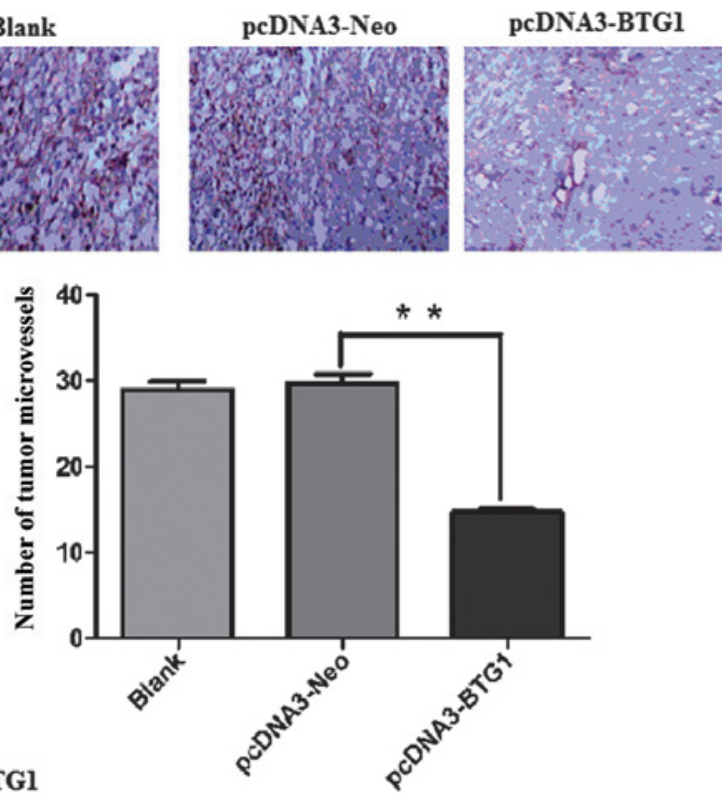

pCDNA3-BTGl
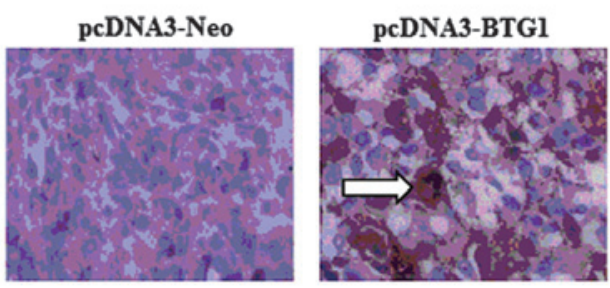

BTG1

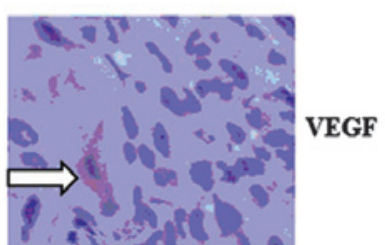

Figure 4. BTG1 negatively affects distant tumor metastasis and angiogenesis in a nude mouse tumor model in vivo. Three groups of MDA-MB-231 cells were injected subcutaneously into nude mice. (A) Hematoxylin and eosin-stained liver tissue from nude mice injected with blank MDA-MB-231 cells. Notable tumor nodule formation was observed in the liver tissue (magnification, x100). (B) Immunohistochemical analysis of angiogenesis (magnification, x100). The numbers of tumor microvessels in the blank and pcDNA3-neo groups were $28.33 \pm 1.53$ and $29.67 \pm 2.08$, respectively. By contrast, in the pcDNA3-BTG1 group, the number of tumor microvessels was $14.00 \pm 1.00\left({ }^{* *} \mathrm{P}<0.01\right)$. (C) In the tumors of mice injected with pcDNA3-BTG1 cells, VEGF expression was lower and BTG1 expression was higher than that in mice injected with the blank and pcDNA3-neo cells (magnification, x200). BTG1, B-cell translocation gene 1; VEGF, vascular endothelial growth factor.

local proteolysis of the ECM, pseudopodial extension and cell migration (20). MMPs, also known as matrixins, are endoproteinases that degrade protein components of the ECM and cause its restoration and reconstruction (21). In the present study, the effect of BTG1 on cell invasion and relative protein expression was examined. Overexpression of BTG1 in breast cancer cells was observed to decrease cell invasion and inhibit MMP-2 and -9 expression. In addition, the adhesion molecule E-cad may have a role in suppressing invasion. E-cad has been suggested to maintain homotypic cell-cell interactions. Furthermore, decreased expression of E-cad has been found to be associated with cancer progression (22). In the present study, overexpression of BTG1 was observed to promote E-cad expression.

In summary, overexpression of BTG1 was found to reduce MMP-2 and -9 expression and promote E-cad expression, leading to decreased breast cancer cell invasion. Of note, the BTG1-induced inhibition of breast cancer cell metastasis in vitro was also confirmed in an animal model in vivo. Significant hepatic metastases were observed in the liver tissue in one out of six mice in the control group, but not in the mice in the BTG1 overexpression group.

Angiogenesis is important for metastasis. A negative correlation has been observed between patient survival and vascularization in several tumor types, including gastric (23), breast (24-26), prostate (27), esophageal (23), vulvar (28) and melanoma (29). High tumor vascularization increases the likelihood of tumor cells entering the circulatory system and metastasizing. It has also been proposed that the newly formed blood vessels may be more permeable to tumor cells and may contribute to new metastases (30). In the present study, the overexpression of BTG1 was found to significantly decrease the number of tumor microvessels in tumor xenografts. Furthermore, expression of BTG1 was observed to be inversely correlated with the expression of VEGF. These results suggest that overexpression of BTG1 may inhibit angiogenesis through downregulating VEGF expression, thereby inhibiting tumor metastasis.

In conclusion, the present study investigated the role of BTG1 in breast cancer metastasis and showed that 
overexpression of BTG1 inhibited breast cancer cell adhesion, migration and invasion, and inhibited in vivo distant tumor metastasis and angiogenesis in a nude mouse tumor model. These findings provide novel insight into the role of BTG1 in breast cancer and may have important implications in the development of targeted therapies for breast cancer.

\section{Acknowledgements}

This study was supported by the National Natural Science Foundation of China (no. 81001185), the Universities Natural Science Foundation of Jiangsu (no. 10KJB310011) and a Project Funded by the Priority Academic Program Development of Jiangsu Higher Education Institutions.

\section{References}

1. Mehlen P and Puisieux A: Metastasis: a question of life or death. Nat Rev Cancer 6: 449-458, 2006.

2. O'Shaughnessy J: Extending survival with chemotherapy in metastatic breast cancer. Oncologist 10 (Suppl 3): 20-29, 2005.

3. Jemal A, Bray F, Center MM, Ferlay J, Ward E and Forman D: Global cancer statistics. CA Cancer J Clin 61: 69-90, 2011.

4. Chang EL and Lo S: Diagnosis and management of central nervous system metastases from breast cancer. Oncologist 8: 398-410, 2003

5. Lassman AB and DeAngelis LM: Brain metastases. Neurol Clin 21: 1-23, 2003.

6. Rouault JP, Rimokh R, Tessa C, Paranhos G, Ffrench M, Duret L, Garoccio M, Germain D, Samarut J and Magaud JP: BTG1, a member of a new family of antiproliferative genes. EMBO J 11: 1663-1670, 1992.

7. Berthet C, Guéhenneux F, Revol V, Samarut C, Lukaszewicz A, Dehay C, Dumontet C, Magaud JP and Rouault JP: Interaction of PRMT1 with BTG/TOB proteins in cell signalling: molecular analysis and functional aspects. Genes Cells 7: 29-39, 2002.

8. Matsuda S, Rouault J, Magaud J and Berthet C: In search of a function for the TIS21/PC3/BTG1/TOB family. FEBS Lett 497: $67-72,2001$

9. Bakker WJ, Blázquez-Domingo M, Kolbus A, Besooyen J, Steinlein P, Beug H, Coffer PJ, Löwenberg B, von Lindern M and van Dijk TB: FoxO3a regulates erythroid differentiation and induces BTG1, an activator of protein arginine methyl transferase 1. J Cell Biol 164: 175-184, 2004.

10. Hata K, Nishijima K and Mizuguchi J: Role for Btg1 and Btg2 in growth arrest of WEHI-231 cells through arginine methylation following membrane immunoglobulin engagement. Exp Cell Res 313: 2356-2366, 2007.

11. Corjay MH, Kearney MA, Munzer DA, Diamond SM and Stoltenborg JK: Antiproliferative gene BTG1 is highly expressed in apoptotic cells in macrophage-rich areas of advanced lesions in Watanabe heritable hyperlipidemic rabbit and human. Lab Invest 78: 847-858, 1998.

12. Lee H, Cha S, Lee MS, Cho GJ, Choi WS and Suk K: Role of antiproliferative B cell translocation gene-1 as an apoptotic sensitizer in activation-induced cell death of brain microglia. J Immunol 171: 5802-5811, 2003.
13. Rodier A, Marchal-Victorion S, Rochard P, Casas F, Cassar-Malek I, Rouault JP, Magaud JP, Mason DY, Wrutniak C and Cabello G: BTG1: a triiodothyronine target involved in the myogenic influence of the hormone. Exp Cell Res 249: 337-348, 1999.

14. Cho JW, Kim JJ, Park SG, Lee DH, Lee SC, Kim HJ, Park BC and Cho S: Identification of B-cell translocation gene 1 as a biomarker for monitoring the remission of acute myeloid leukemia. Proteomics 4: 3456-3463, 2004.

15. Poste $\mathrm{G}$ and Fidler IJ: The pathogenesis of cancer metastasis. Nature 283: 139-146, 1980.

16. Leber MF and Efferth T: Molecular principles of cancer invasion and metastasis (review). Int J Oncol 34: 881-895, 2009.

17. Woodhouse EC, Chuaqui RF and Liotta LA: General mechanisms of metastasis. Cancer 80 (8 Suppl): 1529-1537, 1997.

18. Silletti S, Paku S and Raz A: Tumor cell motility and metastasis: Autocrine motility factor as an example of ecto/exoenzyme cytokines. Pathol Oncol Res 3: 230-254, 1997.

19. Li HZ, Gao Y, Zhao XL, Liu YX, Sun BC, Yang J and Yao Z: Effects of raf kinase inhibitor protein expression on metastasis and progression of human breast cancer. Mol Cancer Res 7: 832-840, 2009.

20. Liotta LA and Stracke ML: Tumor invasion and metastases: biochemical mechanisms. Cancer Treat Res 40: 223-238, 1988.

21. Trojanek J: Matrix metalloproteinases and their tissue inhibitors. Postepy Biochem 58: 353-362, 2012 (In Polish).

22. Bussemakers MJ, van Moorselaar RJ, Giroldi LA, Ichikawa T, Isaacs JT, Takeichi M, Debruyne FM and Schalken JA: Decreased expression of E-cadherin in the progression of rat prostatic cancer. Cancer Res 52: 2916-2922, 1992.

23. Tanigawa $N$, Amaya $H$, Matsumura $M$ and Shimomatsuya $T$ : Association of tumour vasculature with tumour progression and overall survival of patients with non-early gastric carcinomas. Br J Cancer 75: 566-571, 1997.

24. Gasparini G: Clinical significance of the determination of angiogenesis in human breast cancer: update of the biological background and overview of the Vicenza studies. Eur J Cancer 32A: 2485-2493, 1996.

25. Karaiossifidi H, Kouri E, Arvaniti H, Sfikas S and Vasilaros S: Tumor angiogenesis in node-negative breast cancer: relationship with relapse free survival. Anticancer Res 16: 4001-4002, 1996

26. Heimann R, Ferguson D, Powers C, Recant WM, Weichselbaum RR and Hellman S: Angiogenesis as a predictor of long-term survival for patients with node-negative breast cancer. J Natl Cancer Inst 88: 1764-1769, 1996.

27. Silberman MA, Partin AW, Veltri RW and Epstein JI: Tumor angiogenesis correlates with progression after radical prostatectomy but not with pathologic stage in Gleason sum 5 to 7 adenocarcinoma of the prostate. Cancer 79: 772-779, 1997.

28. Obermair A, Kohlberger P, Bancher-Todesca D, Tempfer C, Sliutz G, Leodolter S, Reinthaller A, Kainz C, Breitenecker G and Gitsch G: Influence of microvessel density and vascular permeability factor/vascular endothelial growth factor expression on prognosis in vulvar cancer. Gynecol Oncol 63: 204-209, 1996

29. Srivastava A, Laidler P, Davies RP, Horgan K and Hughes LE: The prognostic significance of tumor vascularity in intermediate-thickness (0.76-4.0 mm thick) skin melanoma. A quantitative histologic study. Am J Pathol 133: 419-423, 1988.

30. Denijn M and Ruiter DJ: The possible role of angiogenesis in the metastatic potential of human melanoma. Clinicopathological aspects. Melanoma Res 3: 5-14, 1993. 